\title{
HAUSDORFF DIMENSION OF HARMONIC MEASURES ON NEGATIVELY CURVED MANIFOLDS
}

\author{
YURI KIFER AND FRANCOIS LEDRAPPIER
}

\begin{abstract}
We show by probabilistic means that harmonic measures on manifolds, whose curvature is sandwiched between two negative constants have positive Hausdorff dimensions. A lower bound for harmonic measures of open sets is derived, as well. We end with the results concerning the Hausdorff dimension of harmonic measures on universal covers of compact negatively curved manifolds.
\end{abstract}

\section{INTRODUCTION}

Let $M$ be a complete, simply connected $m$-dimensional Riemannian manifold with nonpositive sectional curvatures and the Laplace-Beltrami operator

$$
\Delta=\operatorname{divgrad}=\frac{1}{\sqrt{g}} \sum_{1 \leq i, j \leq m} \frac{\partial}{\partial x_{i}}\left(\sqrt{g} g^{i j} \frac{\partial}{\partial x_{j}}\right)
$$

where $g=\operatorname{det}\left(g_{i j}\right),\left(g^{i j}\right)=\left(g_{i j}\right)^{-1}$, and $d s^{2}=\sum_{i \leq i, j \leq m} g_{i j} d x^{i} d x^{j}$ is the metric form on $M$. The $C^{2}$ functions $h$ on $M$ satisfying $\Delta h=0$ are called harmonic. There is a natural geometric compactification of any complete simple connected manifold $M$ of nonpositive curvature by means of the sphere at infinity $S(\infty)$ which is the set of classes of asymptotic geodesics. It is known (see, for instance, [AS] or [Ki]) that under certain assumptions on the curvature of $M$, in particular when all sectional curvatures are sandwiched between two negative constants, any harmonic function $h$ having a continuous extension $f$ to $S(\infty)$ can be represented in the form

$$
h(x)=\int_{S(\infty)} f(\zeta) P(x, d \zeta)
$$

where $P(x, \cdot), x \in M$, are, so-called, harmonic measures. Moreover, any bounded harmonic function can be also represented in the form (1.2) with $f$ being a Borel function on $S(\infty)$. The representation (1.2) is unique for the continuous harmonic functions case and it is unique up to sets of harmonic measures zero for bounded harmonic functions case.

Received by the editors February 17, 1988 and, in revised form, July 22, 1988.

1980 Mathematics Subject Classification (1985 Revision). Primary 58G32.

The first author was partly supported by the U.S. Army Research Office through the Mathematical Sciences Institute of Cornell University. 
For any Borel set $U \subset S(\infty), P(x, U)$ is a harmonic function in $x$ and so by the Harnack inequality (see [Ki])

$$
C^{-1}<P(x, U) / P(y, U) \leq C
$$

when $y \in B_{x}(r)=\{z: \operatorname{dist}(z, x)<r\}$ with $C>0$ independent of $U, x, y$ and depending only on $r$. This implies that all measures $P_{x}, x \in M$, are equivalent, and so these measures form one class of equivalent measures called the harmonic class.

On the other hand, given $x \in M$ each vector $\xi \in S_{x} M=\left\{\eta \in T_{x} M:\|\eta\|=\right.$ $1\}$, where $T_{x} M$ is the tangent space at $x$ and $\|\cdot\|$ is the Riemannian norm there, corresponds uniquely to a point $\zeta_{\xi} \in S(\infty)$ so that $\xi$ is the tangent vector of the geodesic $\gamma(t)$ satisfying $\gamma(0)=x$ and $\lim _{t \rightarrow \infty} \gamma(t)=\zeta_{\xi}$. This yields the map $\Phi_{x}: S_{x} M \rightarrow S(\infty)$ which maps the Lebesgue measure on $S_{x} M$ to some measure $\nu_{x}$ on $S(\infty)$. If $M$ is a cover of a compact manifold of negative curvature then it follows from the absolute continuity of foliations of the geodesic flow (see $\left[\mathrm{Le}_{1}\right]$ ) that all measures $\nu_{x}, x \in M$, are equivalent which determines another class of measures called the geodesic class. D. Sullivan conjectured that for covers of compact negatively curved manifolds measures from the harmonic and geodesic classes are mutually singular unless the manifold has constant curvature. In two dimensions this was shown to be true in $[\mathrm{Ka}]$ and [Le $\mathrm{L}_{1}$.

Since harmonic measures may be singular with respect to the geodesic parametrization it is natural to employ the Hausdorff dimension machinery which enables one to study sets of zero measure. As usual, the Hausdorff dimension of a measure is defined as the infimum of Hausdorff dimensions of sets of full measure where by the Hausdorff dimension $H d_{x}(U)$ of a set $U \subset S(\infty)$ relative to $x \in M$ we mean the Hausdorff dimension of the set $\Phi_{x}^{-1}(U)$ in $S_{x} M$.

In this paper employing the probabilistic approach from [Ki] we shall show that under the assumptions which cover the case of sectional curvatures sandwiched between two negative constants harmonic measures have positive Hausdorff dimensions. By the way we obtain also that these measures are continuous, i.e., they have no atoms. We shall also give some lower bound for harmonic measures of intersections of cones with $S(\infty)$ which improves the known result that harmonic measures of open sets are positive. In the concluding section we discuss Hausdorff dimensions of harmonic measures for universal covers of compact manifolds of negative curvature which involves some ergodic theory machinery. We show in the two-dimensional case that the Hausdorff dimension of harmonic measures equals the dimension of $S(\infty)$ if and only if the measures from the geodesic and the harmonic classes are equivalent which means in this case that the curvature is constant.

\section{Preliminaries}

By the Cartan-Hadamard theorem the exponential map $\exp _{x}: T_{x} M \rightarrow M$ based at any point $x \in M$ is a diffeomorphism of the tangent space $T_{x} M$ at 
$x$ onto $M$. This enables one to introduce a global system of geodesic polar coordinates by assigning to each $z \in M$ a pair $(r, \xi)$ where $r=\operatorname{dist}(x, z), \xi \in$ $S_{x} M, z=\exp _{x}(r \xi)$, and dist is the abbreviation for the Riemannian distance.

As in [Ki] we shall work under the following assumptions.

Assumption $M_{1}$ (bounded geometry). There exist $C_{1}, \delta_{1}>0$ such that any open geodesic ball $B_{x}\left(\delta_{1}\right)$ of radius $\delta_{1}$ centered at an arbitrary $x \in M$ admits a chart $\psi_{x}: B_{x}\left(\delta_{1}\right) \rightarrow R^{m}$ satisfying a uniform quasi-isometry condition

$$
C_{1}^{-1} \operatorname{dist}(y, z) \leq\left|\psi_{x}(y)-\psi_{x}(z)\right| \leq C_{1} \operatorname{dist}(y, z) \text { for any } y, z \in B_{x}\left(\delta_{1}\right) \text {. }
$$

Assumption $M_{2}$ (exponential divergence of geodesics). There exists a number $\alpha_{0}>0$ such that for any point $x \in M$ given tangent vectors $\xi_{1}, \xi_{2} \in S_{x} M$ and numbers $r_{2} \geq r_{1}>0$ one has

$$
e^{\alpha_{0}\left(r_{2}-r_{1}\right)} \leq \frac{\operatorname{dist}_{x}^{\left(r_{2}\right)}\left(\exp _{x} r_{2} \xi_{1}, \exp _{x} r_{2} \xi_{2}\right)}{\operatorname{dist}_{x}^{\left(r_{1}\right)}\left(\exp _{x} r_{1} \xi_{1}, \exp _{x} r_{1} \xi_{2}\right)} \leq e^{\left(x_{0}^{-1}\left(r_{2}-r_{1}\right)\right.}
$$

where $\operatorname{dist}_{x}^{(r)}(\cdot, \cdot)$ is the relative distance on the sphere $S_{x}(r)$ of radius $r$ centered at $x$.

Remark 2.1. It follows from the Rauch comparison theorem (see [CE]) that Assumptions $M_{1}$ and $M_{2}$ are satisfied if all sectional curvatures of $M$ are sandwiched between two negative constants.

We also need

Assumption $\Delta$. There exists a constant $C_{2}>0$ such that for any $x_{0} \in M$ the function $\rho_{x_{0}}(x)=\operatorname{dist}\left(x_{0}, x\right)$ of $x$ satisfies

$$
\Delta \rho_{x_{0}}(x) \geq C_{2}^{-1} \text { for all } x \in M
$$

and

$$
\Delta \rho_{x_{0}}(x) \leq C_{2} \text { provided } \rho_{x_{0}}(x) \geq \frac{1}{2} .
$$

Remark 2.2. By Bishop's comparison theorem (see [Ch]) Assumption $\Delta$ will be satisfied if all sectional curvatures of $M$ are sandwiched between two negative constants. In fact some pieces of zero curvature may be permitted. For instance, if $M$ is the universal cover of a compact manifold $N$ then Assumptions $M_{1}$, $M_{2}$, and $\Delta$ will be satisfied if the geodesic flow on $N$ is an Anosov flow. The latter holds true if, for example, $N$ has nonpositive curvature which is negative outside of a simply connected set (see Additional Remark at the end of [Ki]).

The Laplace-Beltrami operator $\Delta$ generates a diffusion process $X(t)$ (see [IW]), called the Brownian motion on $M$, in the sense that the transition probability $P(t, x, \Gamma)=P_{x}\{X(t) \in \Gamma\}$ satisfies

$$
\partial P / \partial t=\Delta P,\left.\quad P\right|_{t=0}=\chi_{\Gamma}
$$


where $P_{x}\{\cdot\}$ denotes the probability of the event in brackets provided $X(0)=x$ and $\chi_{\Gamma}(x)=1$ if $x \in \Gamma$ and $\chi_{\Gamma}(x)=0$ if $x \notin \Gamma$.

Consider the Brownian motion $X(t)$ in the geodesic polar coordinates with the pole $x$ as a pair $X(t)=\left(R_{x}(t), \Xi_{x}(t)\right)$ where $R_{x}(t)=\operatorname{dist}(X(t), x), \Xi_{X}(t) \in$ $T_{x} M$, and $X(t)=\exp _{x}\left(R_{x}(t) \Xi_{x}(t)\right)$. Let also $\tau_{x}(r)=\inf \left\{t \geq 0: X(t) \in \dot{S}_{x}(r)\right\}$ is the first hitting time by $X(t)$ of the geodesic sphere $S_{x}(r)$ of radius $r$ centered at $x$.

Lemma 2.1. (a) With probability one

$$
R_{x}(t) \geq R_{x}(0)+\sqrt{2} w(t)+C_{2}^{-1} t
$$

and if $\operatorname{dist}\left(x, R_{x}(0)\right)>\frac{1}{2}$ and $0 \leq t<\tau_{x}\left(\frac{1}{2}\right)$ then also

$$
R_{x}(t) \leq R_{x}(0)+\sqrt{2} w(t)+C_{2} t
$$

where $w(t)$ is the one-dimensional Wiener process starting at zero.

(b) There exists a number $\alpha_{1}>0$ such that for any $x \in M$ and $t>0$,

$$
P_{x}\left\{\tau_{x}(1) \leq t\right\} \leq \alpha_{1}^{-1} e^{-\alpha_{1} t^{-1}} .
$$

Representing the Laplace-Beltrami operator in geodesic polar coordinates one derives (2.6) and (2.7) from (2.3) and (2.4), respectively, by a comparison argument (see Lemma 2.1 in [Ki]). For the proof of $(b)$ we refer the reader to Lemma 2.2 from [Ki].

We shall also need a version of Harnack's inequality which follows from [Mo].

Lemma 2.2. For any positive $r_{1}<r_{2} \leq \infty$ there exists $C^{r_{1}, r_{2}}>0$ depending only on $r_{2}-r_{1}$ such that for any couple of points $x, y \in M, y \in B_{x}\left(r_{1}\right)$ and for any function $h$ which is harmonic in the ball $B_{x}\left(r_{2}\right)$ of radius $r_{2}$ centered at $x$ one has

$$
h(y) \leq C^{r_{1} \cdot r_{2}} h(x) .
$$

Recall that two geodesic rays $\gamma_{1}$ and $\gamma_{2}$ on $M$ are called asymptotic if $\operatorname{dist}\left(\gamma_{1}(t), \gamma_{2}(t)\right)$ is a bounded function in $t \geq 0$. The asymptote relation is an equivalence relation. The set of equivalence classes of geodesic rays is called the sphere at infinity $S(\infty)$ of $M$. A natural topology on $\bar{M}=M \cup S(\infty)$, called the cone topology, can be described in the following way. Let $\mathscr{C}_{x}(\xi, \delta)$ be the cone about $\xi \in S_{x} M$ of angle $\delta$ with vertex at $x$, i.e.,

$$
\mathscr{C}_{x}(\xi, \delta)=\exp _{x}\left\{\eta \in T_{x} M: L_{x}(\xi, \eta)<\delta\right\}
$$

where $L_{x}$ denotes the angle in $T_{x} M$. Now truncated cones $\mathscr{T}_{x}(\xi, \delta, r)=$ $\mathscr{C}_{x}(\xi, \delta) \backslash \overline{B_{x}(r)}$ together with open geodesic balls $B_{y}(\rho), y \in M$, for a local basis for the cone topology.

The following result is more convenient for our purposes (see version of Lemma 3.1 from [Ki]). 
Lemma 2.3. Suppose that $\infty \geq r_{3}>r_{2}>r_{1} \geq r_{0}>\frac{1}{2}$ and $x \in M, y=$ $\exp _{x}\left(r_{2} \xi\right), \xi \in S_{x} M$. Then

$$
\begin{aligned}
e^{-C_{2}\left(r_{2}-r_{1}\right)}-\left(e^{C_{2}\left(r_{3}-r_{1}\right)}-1\right)^{-1} & \leq P_{y}\left\{\tau_{x}\left(r_{3}\right)>\tau_{x}\left(r_{1}\right)\right\} \\
& \leq\left(e^{C_{2}^{-1}\left(r_{2}-r_{1}\right)}-1\right)^{-1},
\end{aligned}
$$

provided $\left(C_{2}^{-1} t-r_{3}+r_{2}\right)>0$, and

$$
P_{y}\left\{\tau_{x}\left(r_{3}\right)>t\right\} \leq \frac{2 \sqrt{t}}{\sqrt{\pi}\left(C_{2}^{-1} t-r_{3}+r_{2}\right)} e^{-\frac{\left(c_{2}^{-1} t_{t}-r_{3}+r_{2}\right)^{2}}{4 t}},
$$

$$
\begin{aligned}
& Q_{y}\left(x ; r_{0}, r_{1}, r_{3} ; \rho\right) \\
& \quad \stackrel{\operatorname{def}}{=} P_{y}\left\{\sup _{0 \leq t \leq \min \left(\tau_{x}\left(r_{1}\right), \tau_{x}\left(r_{3}\right)\right)} \operatorname{dist}_{x}^{\left(r_{0}\right)}\left(\exp _{x}\left(r_{0} \Xi_{x}(t)\right), \exp _{x}\left(r_{0} \xi\right)\right) \geq \rho\right\} \\
& \quad \leq P_{y}\left\{\tau_{x}\left(r_{3}\right)>t_{0}\right\}+\alpha_{1}^{-1} t_{0}^{2} e^{-\alpha_{1}\left(\alpha_{0} t_{0}-t_{0}^{-1}\right)}
\end{aligned}
$$

where $t_{0}=\sqrt{\rho} e^{\alpha_{0}\left(r_{1}-r_{0}\right) / 2}$.

Proof. The estimate (2.10) follows from Lemma 2.1(a) by a comparison argument (see Lemma 3.1 in [Ki]). The relation (2.11) is an easy consequence of (2.6) since the event $\left\{\tau_{x}\left(r_{3}\right) \geq t\right\}$ can only occur for $X(t)$ starting at $y$ if

$$
r_{2}+\sqrt{2} w(t)+C_{2}^{-1} t<r_{3} \text {. }
$$

But the probability of the last event can be estimated by the right-hand side of (2.11) (see (3.2) in [Ki]).

To prove $(2.12)$ we notice that

$$
Q_{y}\left(x ; r_{0}, r_{1}, r_{3} ; \rho\right) \leq P_{y}\left\{\tau_{x}\left(r_{3}\right)>t_{0}\right\}+\tilde{Q}_{y}
$$

where

$$
\begin{array}{r}
\widetilde{Q}_{y}=P_{y}\left\{\sup _{0 \leq t \leq \min \left(\tau_{x}\left(r_{1}\right), \tau_{x}\left(r_{3}\right)\right)} \operatorname{dist}_{x}^{\left(r_{0}\right)}\left(\exp _{x}\left(r_{0} \Xi_{x}(t)\right), \exp _{x}\left(r_{0} \xi\right)\right) \geq \rho\right. \\
\text { and } \left.\min \left(\tau_{x}\left(r_{1}\right), \tau_{x}\left(r_{3}\right)\right) \leq t_{0}\right\} .
\end{array}
$$

In view of Assumption $M_{2}$ the event in the last probability can only occur if the Brownian motion $X(t)$ will pass the distance of at least $\rho e^{\alpha_{0}\left(r_{1}-r_{0}\right)}$ for the time $t_{0}$ since by the Rauch comparison theorem any smooth curve staying entirely outside of the ball $B_{x}\left(r_{1}\right)$ has the length not less than the relative length on $S_{x}\left(r_{1}\right)$ of its projection to $S_{x}\left(r_{1}\right)$ along geodesic rays emanating from $x$ which by Assumption $M_{2}$ is at least $e^{\alpha_{0}\left(r_{1}-r_{0}\right)}$ times bigger than the length on $S_{x}\left(r_{0}\right)$ of the corresponding projection to $S_{x}\left(r_{0}\right)$. This means that $\tau_{y}\left(l_{0}\right) \leq t_{0}$ where $l_{0}$ is the integral part of $\rho e^{\left(\alpha_{0}\left(r_{1}-r_{0}\right)\right.}$, and so

$$
\widetilde{Q}_{y} \leq P_{y}\left\{\tau_{y}\left(l_{0}\right) \leq t_{0}\right\}
$$


Define the random points $y_{i}, i=0, \ldots, l_{0}-1$, inductively by $y_{0}=y, y_{i+1}=$ $X\left(\tau_{y_{i}}(1)\right)$; then

$$
\tau_{y}\left(l_{0}\right) \geq \sum_{l_{0}-1 \geq i \geq 0} \tau_{y_{i}}(1) .
$$

By (2.8), (2.14), (2.15) and by strong Markov property

$$
\begin{aligned}
\widetilde{Q}_{y} & \leq P_{y}\left\{\sum_{l_{0}-1 \geq i \geq 0} \tau_{y_{i}}(1) \leq t_{0}\right\} \\
& \leq P_{y}\left\{\tau_{y_{i}}(1) \leq t_{0} l_{0}^{-1} \text { for some } i=0, \ldots, l_{0}-1\right\} \\
& \leq \sum_{l_{0}-1 \leq i \leq 0} E_{y} P_{y_{i}}\left\{\tau_{y_{i}}(1) \leq t_{0} l_{0}^{-1}\right\} \\
& \leq l_{0} \sup _{z \in M} P_{z}\left\{\tau_{z}(1) \leq t_{0} l_{0}^{-1}\right\} \\
& \leq l_{0} \alpha_{1}^{-1} e^{-\alpha_{1} l_{0} t_{0}^{-1}},
\end{aligned}
$$

where $E_{y}$ denotes the expectation for the process $X(t)$ starting at $y$. This together with (2.13) and (2.14) yield (2.12).

By (2.6) the radial part $R_{x}(t)$ of the Brownian motion $X(t)$ tends to the infinity almost surely (a.s.). One concludes easily from (2.12) that with probability one the limit

$$
\lim _{r \rightarrow \infty} \Xi_{x}\left(\tau_{x}(r)\right)=\lim _{t \rightarrow \infty} \Xi_{x}(t)=\Xi_{x}(\infty)
$$

exists (see Corollary 3.1 in [Ki]), and so there exists a random point $X_{x}(\infty) \in$ $S(\infty)$ such that

$$
\lim _{r \rightarrow \infty} X\left(\tau_{x}(r)\right)=\lim _{t \rightarrow \infty} X(t)=X_{x}(\infty) \text { a.s. . }
$$

The well-known probabilistic representation of solutions of the Dirichlet problem yields that any harmonic function $h$ satisfies

$$
h(y)=E_{y} h\left(X\left(\tau_{x}(r)\right)\right)
$$

for any $r>0$ and $y \in B_{x}(r)=\{z: \operatorname{dist}(z, x)<r\}$. If $h$ has a continuous extension to $S(\infty)$ then passing in (2.19) to the limit as $r \rightarrow \infty$ we obtain

$$
h(y)=E_{y} f\left(X_{x}(\infty)\right) .
$$

In the other direction this statement is also true, i.e., any continuous on $S(\infty)$ function $f$ gives rise by (2.20) to a harmonic function $h$ continuous in $M \cup$ $S(\infty)$ (see Theorem 3.1 in [Ki]). In view of our definition of harmonic measures $P_{x}$ in the Introduction we conclude that $P_{x}$ is the distribution of $X_{x}(\infty)$. We remark that

$$
\Phi_{x} \Xi_{x}(\infty)=X_{x}(\infty)
$$


where $\Phi_{x}: S_{x} M \rightarrow S(\infty)$ is the natural map defined in the Introduction, and so the distribution of $\Xi_{x}(\infty)$ is also determined by the harmonic measure $P_{x}$ as its image under $\Phi_{x}^{-1}$.

\section{Positivity of the Hausdorff dimensions}

The straightforward definition of the Hausdorff dimension of a set $U \subset S(\infty)$ corresponding to a pole $x$ is

$$
\begin{aligned}
H d_{x}(U) & =\inf \left\{\alpha \geq 0: \lim _{\rho \rightarrow 0} \inf \sum_{i} \varphi_{i}^{\alpha}=0\right\} \\
& =\sup \left\{\alpha \geq 0: \lim _{\rho \rightarrow 0} \inf \sum_{i} \varphi_{i}^{\alpha}=\infty\right\}
\end{aligned}
$$

where the infimum inside the brackets is taken over all countable covers of $U$ by the sets

$$
V_{x}\left(\xi_{i}, \varphi_{i}\right)=\overline{\mathscr{C}_{x}\left(\xi_{i}, \varphi_{i}\right)} \cap S(\infty), \quad \xi_{i} \in S_{x} M, 0 \leq \varphi_{i} \leq \rho,
$$

and, again, $\mathscr{C}_{x}(\xi, \varphi)$ denotes a cone. Next, we define the Hausdorff dimension of any probability measure $m$ on $S(\infty)$ by

$$
H D_{x}(m)=\inf \left\{H d_{x}(U): U \subset S(\infty) \text { and } m(U)=1\right\} \text {. }
$$

Both numbers $H d_{x}(U)$ and $H D_{x}(m)$, in general, may depend on $x$ but if the manifold $M$ has bounded nonpositive curvature then one can see easily that the answer to the question whether these numbers are positive or zero is independent of $x$.

The main result of this section is

Theorem 3.1. Suppose that a complete simply connected Riemannian manifold $M$ of nonpositive curvature satisfies Assumptions $M_{1}, M_{2}$, and $\Delta$. Then there exists a number $\kappa>0$ (depending only on the geometric constants in the above assumptions) and a sequence of numbers $c_{k}>0$ such that

$$
P_{x}\left\{\left\|\Xi_{x}(\infty, \omega)-\xi\right\| \leq \varphi \text { and } \omega \in \Omega_{x}^{(k)}\right\} \leq c_{k} \varphi^{\kappa}
$$

for any $x \in M, \xi \in S_{x} M$, and $\varphi \geq 0$ where $\Omega_{x}^{(k)} \subset \Omega, k=1,2, \ldots, \Omega_{x}^{(k)} \uparrow$ as $k \uparrow \infty$ is an increasing sequence of events such that $P_{x}\left\{\bigcup_{k} \Omega_{x}^{(k)}\right\}=1$.

Before proving this theorem we shall derive

Corollary 3.1. Harmonic measures $P(x, \cdot)$ have no atoms and for any $x \in M$,

$$
H D_{x}(P(x, \cdot)) \geq \kappa
$$

where $\kappa>0$ is the same as in Theorem 3.1.

Proof of Corollary 3.1. Suppose that $P(x, \cdot)$ has an atom at a point $\zeta \in S(\infty)$ such that $\zeta=\lim _{t \rightarrow \infty} \exp _{x}(\xi t)$ for some $\xi \in S_{x} M$. Then

$$
\begin{aligned}
0 & <p=P(x, \zeta) \leq P_{x}\left\{\left\|\Xi_{x}(\infty)-\xi\right\| \leq \varphi\right\} \\
& \leq P_{x}\left\{\left\|\Xi_{x}(\infty, \omega)-\xi\right\| \leq \varphi \text { and } \omega \in \Omega_{x}^{(k)}\right\}+P_{x}\left\{\Omega \backslash \Omega_{x}^{(k)}\right\}
\end{aligned}
$$


for any $\varphi>0$. Choosing first $k$ big enough and then $\varphi>0$ small enough we can make in view of (3.4) the right-hand side of (3.6) arbitrarily small in contradiction with the left-hand side of (3.6). This "no atoms" property follows also from Lemma 7.4 of [AS].

Next, pick up $k_{0}$ so that $P_{x}\left\{\Omega_{x}^{\left(k_{0}\right)}\right\} \geq \frac{1}{2}$ and define the probability measure $\mu_{x}$ on $S(\infty)$ by

$$
\mu_{x}(U)=\left(P_{x}\left\{\boldsymbol{\Omega}_{x}^{\left(k_{0}\right)}\right\}\right)^{-1} P_{x}\left\{X(\infty, \omega) \in U \text { and } \omega \in \Omega_{x}^{\left(k_{0}\right)}\right\} .
$$

Let $\left\{V_{x}\left(\xi_{i}, \varphi_{i}\right), i=1,2, \ldots\right\}$ be a countable cover of a Borel set $U \subset S(\infty)$ satisfying $\mu_{x}(U)=1$ by the sets $V_{x}\left(\xi_{i}, \varphi_{i}\right)$ defined in (3.2). Then by (3.4),

$$
\begin{aligned}
1 & =\mu_{x}\left(\bigcup_{i} V_{x}\left(\xi_{i}, \varphi_{i}\right)\right) \leq \sum_{i} \mu_{x}\left(V_{x}\left(\xi_{i}, \varphi_{i}\right)\right) \\
& \leq 2 \sum_{i} P_{x}\left\{X(\infty, \omega) \in V_{x}\left(\xi_{i}, \varphi_{i}\right) \text { and } \omega \in \Omega_{x}^{\left(k_{0}\right)}\right\} \\
& =2 \sum_{i} P_{x}\left\{\left\|\Xi_{x}(\infty, \omega)-\xi_{i}\right\| \leq \varphi_{i} \text { and } \omega \in \Omega_{x}^{\left(k_{0}\right)}\right\} \\
& \leq 2 c_{k_{0}} \sum_{i} \varphi_{i}^{\kappa},
\end{aligned}
$$

i.e. for any such cover $\sum_{i} \varphi_{i}^{\kappa} \geq\left(2 c_{k_{0}}\right)^{-1}$ which together with Definition (3.1) implies $H d_{x}(U) \geq \kappa$. This being true for any $U$ with $\mu_{x}(U)=1$ yields $H D_{x}\left(\mu_{x}\right) \geq \kappa$. Since, clearly, $\mu_{x}$ is absolutely continuous with respect to $P(x, \cdot)$ then $(3.5)$ follows as well.

For any $x \in M, \xi \in S_{x} M, t>0, \rho>0, a_{2}>a_{1}>0$ define

$$
U_{t}^{a_{1} \cdot a_{2}}(x, \xi, \rho)=\bigcup_{0 \leq k \leq\left[t\left(a_{2}-a_{1}\right) \rho^{-1}\right]+1} B_{z_{k}}(2 \rho)
$$

where the balls $B_{z_{k}}(2 \rho)$ of radius $2 \rho$ are centered at the points

$$
z_{k}=\exp _{x}\left(\left(a_{1} t+k \rho\right) \xi\right)
$$

and [.] denotes the integral part. The main step in the proof of Theorem 3.1 is

Lemma 3.1. For any $\alpha, \delta>0$ there exists $\beta=\beta(\alpha, \delta)>0$ such that

$$
P_{x}\left\{X(t) \in U_{t}^{a_{1}, a_{2}}\left(x, \Xi_{x}(\infty), t^{\alpha}\right)\right\} \geq 1-\beta^{-1} t^{-\beta}
$$

for all $t>0$ and $x \in M$ provided $a_{1} \leq C_{2}^{-1}-\delta \leq C_{2}+\delta \leq a_{2}$, and

$$
P_{z}\left\{\operatorname{sup~dist}_{t>0}^{(\rho)}\left(\exp _{x}\left(\rho \Xi_{x}(t), z\right)\right) \leq\left(\frac{\rho}{a_{2}}\right)^{\alpha}\right\} \geq 1-\beta^{-1} \rho^{-\beta}
$$

for any $\rho>0$ and $z \in S_{x}(\rho)$.

Proof. From Lemma 2.1(a) and our assumption on $a_{1}, a_{2}$ it follows that

$$
\left(a_{1}+\delta\right) t+\sqrt{2} w(t) \leq R_{x}(t)-R_{x}(0) \leq\left(a_{2}-\delta\right) t+1+\sqrt{2} w(t),
$$


and so

$$
\begin{aligned}
P_{x}\left\{a_{1} t\right. & \left.\leq R_{x}(t) \leq a_{2} t\right\} \geq P\{\sqrt{2}|w(t)| \leq \delta t-1\} \\
& =1-2 \int_{\delta t-1}^{\infty} \frac{1}{2 \sqrt{\pi t}} e^{\frac{u^{2}}{4 t}} d u \geq 1-\frac{4 \sqrt{t}}{(\delta t-1) \sqrt{\pi}} e^{-\frac{(\delta t-1)^{2}}{4 t}} .
\end{aligned}
$$

Next, pick up a point $z=(\rho, \xi), \xi \in S_{x} M$ with $\rho \geq \rho_{0}$ large enough. For $n=1,2, \ldots$ define

$$
\begin{gathered}
W_{n}(z)=\left\{\eta \in S_{x} M: \operatorname{dist}_{x}^{(\rho)}\left(\exp _{x}(\rho \xi), \exp _{x}(\rho \eta)\right) \leq\left(1-2^{-n}\right)\left(\frac{\rho}{a_{2}}\right)^{\alpha}\right\}, \\
l_{n}^{\varepsilon}(z)=\bigcup_{\eta \in W_{n}(z)} \exp _{x}\left(r_{\varepsilon}(n) \eta\right),
\end{gathered}
$$

and

$$
D_{n}^{\varepsilon}(z)=\bigcup_{-\varepsilon \ln \rho-(n-1) \leq t \leq \varepsilon \ln \rho+n} \bigcup_{\eta \in W_{n}(z)} \exp _{x}\left(\left(r_{\varepsilon}(n)+t\right) \eta\right)
$$

where $r_{\varepsilon}(n)=\rho+\varepsilon(n-1) \ln \rho+n(n-1) / 2$ and, again, $\operatorname{dist}_{x}^{(\rho)}(\cdot, \cdot)$ denotes the relative distance on the sphere $S_{x}(\rho)$. In view of Assumption $M_{2}$ we can choose $\varepsilon>0$ so that for any $\eta_{1}, \eta_{2} \in S_{x} M$ and $u \geq 1$,

$$
\begin{gathered}
\operatorname{dist}_{x}^{(u+\varepsilon \ln \rho)}\left(\exp _{x}\left((u+\varepsilon \ln \rho) \eta_{1}\right), \exp _{x}\left((u+\varepsilon \ln \rho) \eta_{2}\right)\right) \\
\leq\left(\rho / a_{2}\right)^{\alpha / 2} \operatorname{dist}_{x}^{(u)}\left(\exp _{x}\left(u \eta_{1}\right), \exp _{x}\left(u \eta_{2}\right)\right)
\end{gathered}
$$

We also pick up $\rho_{0}>1$ so that

$$
\rho>\varepsilon \ln \rho+2 \text { for any } \rho \geq \rho_{0} .
$$

Put $r_{\varepsilon}(0)=\rho-\varepsilon \ln \rho$ and for $n=1,2, \ldots$ denote

$$
\begin{aligned}
& \partial_{n}^{+}(z)=D_{n}^{\varepsilon}(z) \cap S_{x}\left(r_{\varepsilon}(n+1)\right), \\
& \partial_{n}^{-}(z)=D_{n}^{\varepsilon}(z) \cap S_{x}\left(r_{\varepsilon}(n-1)\right),
\end{aligned}
$$

and

$$
\partial_{n}^{\mathscr{C}}(z)=\partial D_{n}^{\varepsilon}(z) \backslash\left(\partial_{n}^{+}(z) \cup \partial_{n}^{-}(z)\right)
$$

where $\partial U$ denotes the boundary of a set $U$. The following figure 3.1 makes the above notations more transparent.

According to (2.10), for $n=1,2, \ldots$,

$$
\begin{aligned}
& \sup _{y \in S_{x}\left(r_{\varepsilon}(n)\right)} P_{y}\left\{\tau_{x}\left(r_{\varepsilon}(n+1)\right)>\tau_{x}\left(r_{\varepsilon}(n-1)\right)\right\} \\
& \leq e^{-C_{2}^{-1}(n-1)}\left(\rho^{\varepsilon C_{2}^{-1}}-1\right)^{-1} .
\end{aligned}
$$

Let $\tau_{n}=\inf \left\{t \geq 0: X(t) \notin D_{n}^{\varepsilon}(z)\right\}$ be the exit time of the Brownian motion $X(t)$ from $D_{n}^{\varepsilon}(z)$. Then for any $y \in \partial_{n-1}^{+}$if $n>1$ and for $y=z$ if $n=1$ it follows that

$$
\begin{aligned}
& P_{y}\left\{X\left(\tau_{n}\right) \notin \partial_{n}^{+}\right\} \\
& \quad \leq P_{y}\left\{\tau_{x}\left(r_{\varepsilon}(n+1)\right)>\tau_{x}\left(r_{\varepsilon}(n)\right)\right\}+P_{y}\left\{X\left(\tau_{n}\right) \in \partial_{n}^{\mathscr{C}}(x)\right\} .
\end{aligned}
$$




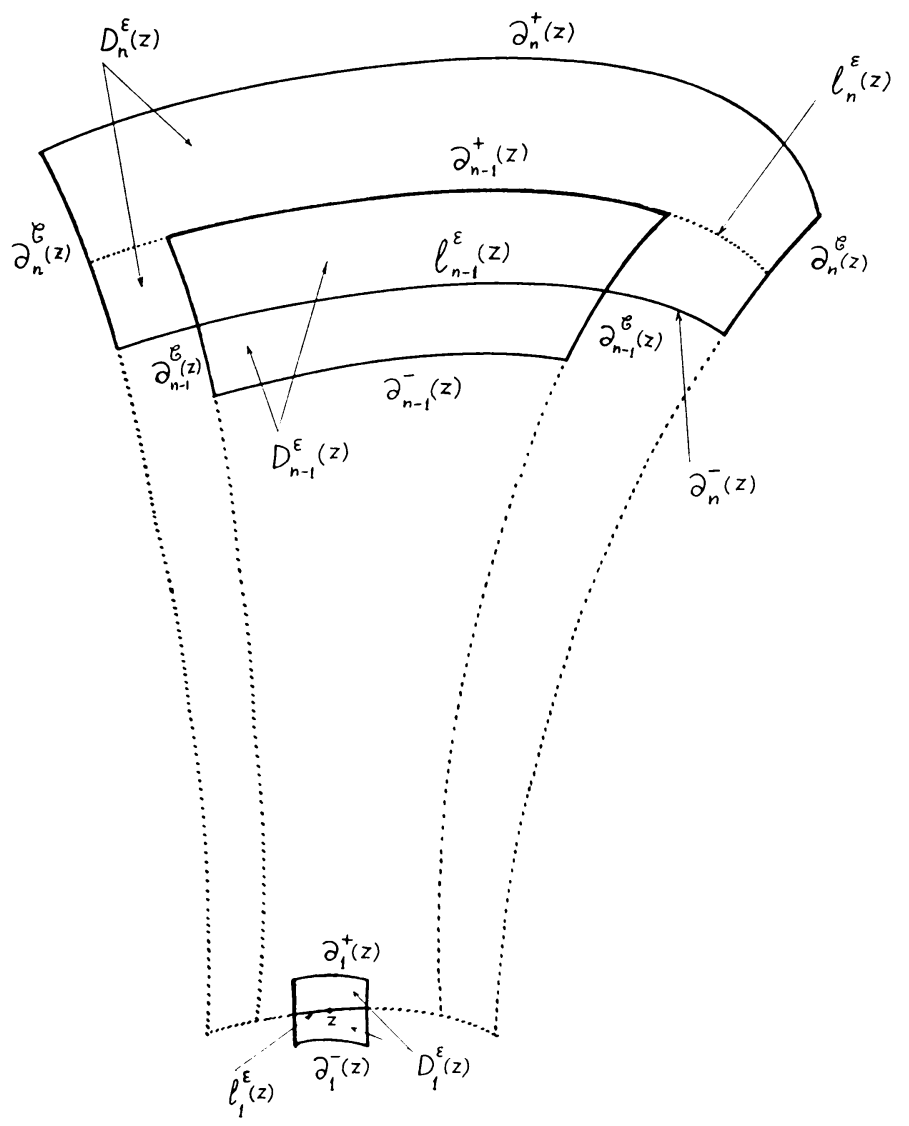

Figure 3.1

By (3.12) and the definition of $D_{1}^{\varepsilon}(z)$,

$$
P_{z}\left\{X\left(\tau_{1}\right) \in \partial_{1}^{\mathscr{F}}(z)\right\} \leq Q_{z}\left(x ; r_{\varepsilon}(0), r_{\varepsilon}(0), r_{\varepsilon}(2) ; \frac{1}{2}\left(\frac{\rho}{a_{2}}\right)^{\alpha / 2}\right) .
$$

Furthermore, by the definition of $D_{n}^{\varepsilon}(z), n=2,3, \ldots$, for any $y \in \partial_{n-1}^{+}$

(3.17) $P_{y}\left\{X\left(\tau_{n}\right) \in \partial_{n}^{\mathscr{\hbar}}(z)\right\} \leq Q_{y}\left(x ; r_{\varepsilon}(1), r_{\varepsilon}(n-1), r_{\varepsilon}(n+1), 2^{-n}\left(\rho / a_{2}\right)^{\alpha}\right)$.

From (2.11), (2.12), and (3.14)-(3.17) we derive that

$$
P_{y}\left\{X\left(\tau_{n}\right) \notin \partial_{n}^{+}\right\} \leq q^{-1} \rho^{-q} e^{-q n}
$$

for some constant $q>0$ independent of $z, y, n$ provided $y=z$ if $n=1$ and $y \in \partial_{n-1}^{+}$if $n>1$. Thus by (3.18) and the strong Markov property of the 
Brownian motion $X(t)$ one has

$$
\begin{aligned}
& P_{z}\left\{\operatorname{dist}_{x}^{(\rho)}\left(\exp _{x}\left(\rho \Xi_{x}(\infty)\right), z\right) \leq\left(\frac{\rho}{a_{2}}\right)^{\alpha}\right\} \\
& \quad \geq P_{z}\left\{\sup _{t \geq 0} \operatorname{dist}_{x}^{(\rho)}\left(\exp _{x}\left(\rho \Xi_{x}(t)\right), z\right) \leq\left(\frac{\rho}{a_{2}}\right)^{\alpha}\right\} \\
& \quad \geq E_{z} \chi_{X\left(\tau_{1}\right) \in \partial_{1}^{+}} E_{X\left(\tau_{1}\right)} \chi_{X\left(\tau_{2}\right) \in \partial_{2}^{+}} E_{X\left(\tau_{2}\right)} \chi_{X\left(\tau_{3}\right) \in \partial_{3}^{+}} \times \cdots \times \\
& \quad \geq 1-P_{z}\left\{X\left(\tau_{1}\right) \notin \partial_{1}^{+}\right\}-\sum_{n=2}^{\infty} \sup _{y \in \partial_{n-1}^{+}} P_{y}\left\{X\left(\tau_{n}\right) \notin \partial_{n}^{+}\right\} \\
& \quad \geq 1-q^{-2} \rho^{-q}
\end{aligned}
$$

implying (3.9), where, as usual, $\chi_{A}=1$ if an event $A$ occurs and $\chi_{A}=0$ for otherwise. Finally, (3.11) and (3.19) together with the Markov property of the process $X(t)$ yield

$$
\begin{aligned}
& P_{x}\left\{X(t) \in U_{t}^{a_{1}, a_{2}}\left(x, \Xi_{x}(\infty), t^{\alpha}\right)\right\} \\
& \geq P_{x}\left\{a_{1} t \leq R_{x}(t) \leq a_{2} t \text { and } \operatorname{dist}_{x}^{\left(R_{x}(t)\right)}\left(\exp _{x}\left(R_{x}(t) \Xi_{x}(\infty)\right), X(t)\right) \leq t^{\alpha}\right\} \\
& \geq \int_{B_{x}\left(a_{2} t\right) \backslash B_{x}\left(a_{1} t\right)} P(t, x, d z) \\
& \times P_{z}\left\{\operatorname{dist}_{x}^{\left(\rho_{x}(z)\right)}\left(\exp _{x}\left(\rho_{x}(z) \Xi_{x}(\infty)\right), z\right) \leq\left(\frac{\rho_{x}(z)}{a_{2}}\right)^{\alpha}\right\} \\
& \geq\left(1-q^{-2} a_{1}^{-q} t^{-q}\right)\left(1-\frac{4 \sqrt{t}}{(\delta t-1)}\right)
\end{aligned}
$$

where $\rho_{x}(z)=\operatorname{dist}(x, z)$ and $P(t, x, \Gamma)=P_{x}\{X(t) \in \Gamma\}$ is the transition probability of the Markov process $X(t)$. This implies (3.8).

Let $p(t, x, y)$ be the transition density of $X(t)$ with respect to the Riemannian volume element $d y$ on $M$, i.e.,

$$
P_{x}\{X(t) \in \Gamma\}=\int_{\Gamma} p(t, x, y) d y .
$$

By the analogy with the usual Laplacian $p(t, x, y)$ is often called the heat kernel since it is the minimal positive fundamental solution of the parabolic equation $\partial u(t, x) / \partial t=\Delta u(t, x)$. We shall need the following result which was proved in [Ki] (see (4.3) there).

Lemma 3.2. There exists a constant $b>0$ such that

$$
p(t, x, y) \leq b^{-1} \min \left(e^{-b t}, e^{-b \operatorname{dist}(x, y)}\right)
$$

for all $x, y \in M$, and $t \geq 1$.

Next, we shall pass to the proof of Theorem 3.1. Fix some $\delta>0$ and $\alpha<1$ and take $\beta=\beta(\alpha, \delta)$ obtained in Lemma 3.1. Put $t_{n}=n^{2 / \beta}$; then (3.8) 
together with the Borel-Cantelli lemma yield that for almost all $\omega \in \Omega$ there exists $n_{x}(\omega)<\infty$ such that $n=n_{x}(\omega)$ is measurable and

$$
X\left(t_{n}, \omega\right) \in U_{t_{n}}^{a_{1} \cdot a_{2}}\left(x, \Xi(\infty, \omega), t_{n}^{\alpha^{x}}\right) \text { if } n \geq n_{x}(\omega) .
$$

Define $\Omega_{x}^{(k)}=\left\{\omega: n_{x}(\omega) \leq k\right\}, k=1,2, \ldots$. By the right-hand side of $(2.2)$ we can choose $L>0$ so that if $\|\xi-\eta\| \leq e^{-L t}$ for $\xi, \eta \in S_{x} M$ then

$$
\operatorname{dist}\left(\exp _{x}\left(\left(a_{2}+1\right) t \xi\right), \exp _{x}\left(\left(a_{2}+1\right) t \eta\right)\right) \leq 1 \text {. }
$$

Thus if $\varphi_{n}=e^{-L t_{n}}$ and $n \geq k$ then by (3.23) and (3.24) for any $\xi \in S_{x} M$

$$
\begin{aligned}
& P_{x}\left\{\|\Xi(\infty, \omega)-\xi\| \leq \varphi_{n} \text { and } \omega \in \Omega_{x}^{(k)}\right\} \\
& \leq P_{x}\left\{\omega: X\left(t_{n}, \omega\right) \in U_{t_{n}}^{a_{1} \cdot a_{2}}\left(x, \xi, t_{n}^{(\alpha}+1\right)\right\} \\
& \leq\left(\sup _{y: \operatorname{dist}(x, y) \geq a_{1} t_{n}-2 t_{n}^{\prime \prime}-1} p\left(t_{n}, x, y\right)\right) \\
& \times \operatorname{vol}\left(U_{t_{n}}^{a_{1}, a_{2}}\left(x, \xi, t_{n}^{\mathrm{\alpha}}+1\right)\right) \text {. }
\end{aligned}
$$

From Assumption $M_{2}$ it follows that the volume of $U_{t_{n}}^{a_{1}, a_{2}}\left(x, \xi, t_{n}^{\alpha^{k}}+1\right)$ is bounded by $d^{-1} e^{d t_{n}^{\prime \prime}}$ for some constant $d>0$ independent of $x, \xi$, and $n$. Since $\alpha<1$ then this together with (3.22) and (3.25) give for $n \geq k$.

$$
\begin{aligned}
& P_{x}\left\{\|\Xi(\infty, \omega)-\xi\| \leq \varphi_{n} \text { and } \omega \in \Omega_{x}^{(k)}\right\} \\
& \leq(b d)^{-1} e^{d t_{n}^{\prime \prime}-b t_{n}} \leq K e^{-b t_{n} / 2}=K \varphi_{n}^{b / 2 L}
\end{aligned}
$$

for some $K>0$ independent of $n$. Since

$$
\frac{\ln \varphi_{n+1}}{\ln \varphi_{n}}=\frac{t_{n+1}}{t_{n}} \rightarrow 1
$$

as $n \rightarrow \infty$ then (3.26) yields (3.4).

\section{A LOWER BOUND}

The uniqueness of the representation (1.2) for harmonic functions $h$ by means of continuous functions on $S(\infty)$ implies that harmonic measures of open subsets of $S(\infty)$ are positive. The following result improves this statement.

Theorem 4.1. Suppose that a complete simply connected Riemannian manifold $M$ of nonpositive curvature satisfies Assumptions $M_{1}, M_{2}$, and $\Delta$ Then there exists a constant $N>0$ such that

$$
P\left(x, \mathscr{C}_{x}(\xi, \varphi) \cap S(\infty)\right)=P_{x}\left\{\left\|\Xi_{x}(\infty)-\xi\right\| \leq \varphi\right\} \geq N^{-1} \varphi^{N}
$$

for all $x \in M, \xi \in S_{x} M$, and $\varphi \geq 0$.

Remark 4.1. By Proposition 2.1 of [Y], (4.1) implies $H D_{x}(P(x, \cdot)) \leq N$. On the other hand, it is easy to see that (4.1) satisfied for all $x, \xi, \varphi$ yields $N \geq$ 
$m-1$ where $m=\operatorname{dim} M$. So this does not give more than the obvious estimate $H D_{x}(P(x, \cdot)) \leq m-1$.

For any $x \in M, \xi \in S_{x} M$, and $r, \rho>0$ denote

$$
Z_{x}(\xi, r ; \rho)=\left\{y \in S_{x}(r): \operatorname{dist}_{x}^{(r)}\left(y, \exp _{x}(r \xi)\right)<\rho\right\} .
$$

We shall need the following auxiliary result:

Lemma 4.1. There exists $q_{1}>0$ such that

$$
P_{x}\left\{X\left(\tau_{x}(2)\right) \in Z_{x}(\xi, 2,1)\right\} \geq q_{1}
$$

and

$$
\inf _{y \in Z_{x}(\xi, n ; 1)} P_{y}\left\{X\left(\tau_{x}(n+1)\right) \in Z_{x}(\xi, n+1,1)\right\} \geq q_{1}
$$

for any $x \in M, \xi \in S_{x} M$, and $n=2,3, \ldots$.

Proof. Set

$$
\Psi_{x}(\xi, r ; \rho)=\left\{\eta \notin S_{x} M: \exp _{x}(r \eta) \in Z_{x}(\xi ; r ; \rho)\right\}
$$

and for $n \geq 2$,

$$
D_{x}(\xi, n)=\bigcup_{n-1<s<n+1} \exp _{x}\left(s \Psi_{x}(\xi, n-1 ; 1)\right) .
$$

Then $D_{x}(\xi, n)$ is a domain with the piecewise smooth boundary and by Assumption $M_{2}$ it follows that

$$
\inf _{z \in \partial D_{x}(\xi, n)} \operatorname{dist}\left(z, Z_{x}(\xi, n ; 1)\right) \geq d_{0}=1-e^{-\alpha_{0}} .
$$

There exists $d_{1}>0, d_{1}<d_{0}$ such that for any $y \in Z_{x}(\xi, n ; 1)$ one can choose a sequence of points $y_{0}=y, y_{1}, \ldots, y_{k}$ such that $y_{k} \notin D_{x}(\xi, n)$, dist $\left(y_{i}, y_{i+1}\right)$ $=d_{1}, i=0, \ldots, k-1$, and

$$
\left(\bigcup_{0 \leq i \leq k} B_{y_{i}}\left(2 d_{1}\right)\right) \cap \partial D_{x}(\xi, n) \subset Z_{x}(\xi, n+1,1) .
$$

The collection of such sequences starting at $y$ we shall denote by $\mathscr{T}_{k}(y)$. Remark that the number $k$ can be fixed independently of $y \in Z_{x}(\xi, n ; 1)$ provided it is big enough.

It is well known that the distributions $P_{z}\left\{X\left(\tau_{z}\left(d_{1}\right)\right) \in \Gamma\right\}, \Gamma \subset S_{z}\left(d_{1}\right)$ have densities $p_{d_{1}}(z, \gamma), \gamma \in S_{z}\left(d_{1}\right)$ with respect to the induced Riemannian volume on $S_{z}\left(d_{1}\right)$ and these densities turn out to be normal derivatives at corresponding points $\gamma \in S_{z}\left(d_{1}\right)$ of the Green function of $\Delta$ in $B_{z}\left(d_{1}\right)$ (see, for instance, [Fr, Chapter 6]). Moreover, it follows from the estimates of $\S 2$ from [Se] that

$$
d_{2} \leq p_{d_{1}}(z, \gamma) \leq d_{2}^{-1}
$$

for all $z \in M$ and $\gamma \in S_{z}\left(d_{1}\right)$ where $d_{2}>0$ depends only on the ellipticity constant of the operator $\Delta$ which is determined by $C_{1}$ in Assumption $M_{1}$. 
It follows easily from (4.4) and (4.6) that the probability of the sequence of random points

$$
y_{0}=y, \quad y_{1}=X\left(\tau_{y_{0}}\left(d_{1}\right)\right), \quad y_{2}=X\left(\tau_{y_{1}}\left(d_{1}\right)\right), \ldots, y_{k}=X\left(\tau_{y_{k-1}}\left(d_{1}\right)\right)
$$

belonging to the collection $\mathscr{T}_{k}(y)$ is bounded away from zero by a constant $q_{1}>0$ independent of $x \in M, \xi \in S_{x} M, n \geq 2$, and $y \in Z_{x}(\xi, n ; 1)$. Thus

$$
\inf _{y \in Z_{x}(\xi, n ; 1)} P_{y}\left\{X\left(\sigma_{x}(\xi, n)\right) \in Z_{x}(\xi, n+1,1)\right\} \geq q_{1}
$$

where $\sigma_{x}(\xi, n)=\inf \left\{t \geq 0: X(t) \notin D_{x}(\xi, n)\right\}$ which, clearly, yields (4.3).

As above, the arguments from $\S 2$ of [Se] imply that the density $p_{2}(x, \gamma)$ of the distribution $P_{x}\left\{X\left(\tau_{x}(2)\right) \in d \gamma\right\}$ on $S_{x}(2)$ is also sandwiched between two positive constants independent of $x$ and $\gamma$ and (4.2) follows.

Next, for $x \in M, \xi \in S_{x} M$, and $\varphi>0, \varphi \leq \pi / 2$ define

$$
K_{x}(\xi, \varphi)=\min \left\{n \geq 1: \mathscr{C}_{x}(\xi, \varphi) \cap S_{x}(n) \supset Z_{x}(\xi, n ; 1)\right\} .
$$

We also need

Lemma 4.2. For any $x, \xi$, and $\varphi$ as above and $K=K_{x}(\xi, \varphi)$,

$$
\inf _{z \in Z_{x}(\xi, K+1 ; 1)} P_{z}\left\{X(t) \in \mathscr{C}_{x}(\xi, \varphi) \text { for all } t \geq 0\right\} \geq q_{2}
$$

where $q_{2}>0$ is independent of $x, \xi$, and $\varphi$.

Proof. Choose an integer $n_{0}=\rho>K$ in (3.9) big enough so that $1-\beta^{-1} n_{0}^{-\beta} \geq$ $\frac{1}{2}$ and $\mathscr{C}_{x}(\xi, \varphi) \cap S_{x}\left(n_{0}\right) \supset Z_{x}\left(\xi, n_{0} ;\left(n_{0} / a_{2}\right)^{\alpha}+1\right)$. Then by (3.9) for any $z \in Z_{x}\left(\xi, n_{0} ; 1\right)$

$$
P_{z}\left\{X(t) \in \mathscr{C}_{X}(\xi, \varphi) \text { for all } t \geq 0\right\} \geq \frac{1}{2} .
$$

By (4.7), (4.9), and the strong Markov property of the Brownian motion $X(t)$ for any $z \in Z_{x}(\xi, K+1,1)$,

$$
\begin{aligned}
& P_{z}\left\{X(t) \in \mathscr{C}_{X}(\xi, \varphi) \text { for all } t \geq 0\right\} \\
& \geq E_{z} \prod_{n_{0} \geq l \geq K+1} \chi_{X\left(\sigma_{x}(\xi, l)\right) \in Z_{x}(\xi, l, 1)} \\
& \times P_{X\left(\tau_{x}\left(n_{0}\right)\right)}\left\{X(t) \in \mathscr{C}_{X}(\xi, \varphi) \text { for all } t \geq 0\right\} \\
& \geq \frac{1}{2} q_{1}^{n_{0}-K} \geq q_{2}
\end{aligned}
$$

where $q_{2}>0$ is independent of $x, \xi$, and $\varphi$ since, clearly, $n_{0}-K$ is bounded by a constant depending only on $\alpha_{0}$ in Assumption $M_{2}$.

Proof of Theorem 4.1. In the same way as in (4.10) we derive from (4.2), (4.3), (4.8), and the strong Markov property of $X(t)$ that

$$
\begin{aligned}
& P_{x}\left\{\left\|\Xi_{x}(\infty)-\xi\right\| \leq \varphi\right\} \\
& \quad \geq E_{x} \prod_{K+1 \geq l \geq 2} \chi_{X\left(\tau_{x}(l)\right) \in Z_{x}(\xi, l, 1)} \\
& \quad \times P_{X\left(\tau_{x}(K+1)\right)}\left\{X(t) \in \mathscr{C}_{x}(\xi, \varphi) \text { for all } t \geq 0\right\} \geq q_{2} q_{1}^{K+1} .
\end{aligned}
$$


By (2.2) and the definition of $K=K_{x}(\xi, \varphi)$ it follows that

$$
e^{-\alpha_{0} K} \leq \varphi \leq e^{-\alpha_{0}(K-1)}
$$

which together with (4.11) yield

$$
P_{x}\left\{\left\|\Xi_{x}(\infty)-\xi\right\| \leq \varphi\right\} \geq q_{2} q_{1}^{2} \varphi^{\frac{1}{n_{0}} \ln \frac{1}{q_{1}}}
$$

proving (4.1).

\section{HARMONIC MEASURES ON COVERING SPACES}

In this section we assume that $M$ is the universal cover of a compact connected Riemannian manifold $N$ of negative curvature, i.e. $N=M / \Gamma$ with $\Gamma$ being the fundamental group of $N$ acting isometrically on $M$. In this situation one may expect that the Hausdorff dimensions $H D_{x}(P(x, \cdot))$ of harmonic measures are independent of $x \in M$ and that the corresponding number reflects some geometrical properties of $N$. However, we are able to prove this only when the dimension $m$ of $M$ is 2 .

Theorem 5.1. Let $N$ be a compact connected Riemannian surface with negative curvature. Then there exists a positive number $\kappa \leq 1$ such that for all points $x$ in the universal cover $M$ of $N$ and for $\Phi_{x}^{-1} P(x, \cdot)$-almost all $\xi \in S_{x} M$,

$$
\lim _{\varphi \rightarrow 0} \frac{\ln \left(P_{x}\left\{\left\|\Xi_{x}(\infty, \omega)-\xi\right\| \leq \varphi\right\}\right)}{\ln \varphi}=\kappa
$$

where $\Phi_{x}$ performs the natural one-to-one correspondence between points and measures on $S_{x} M$ and points and measures on $S(\infty)$ along geodesic rays emanating from $x$. Moreover $\kappa=1$ if and only if the curvature is constant.

Corollary 5.1. For all $x \in M$ one has

$$
H D_{x} P(x, \cdot)=\inf \left\{H d_{x}(U): U \subset S(\infty) \text { and } P(x, U)>0\right\}=\kappa .
$$

This result follows immediately from (5.1) and Proposition 2.1 of [Y], the argument being similar to (3.7).

Next, we proceed to a sketch of the proof of Theorem 5.1. The full details and applications will appear in $\left[\mathrm{Le}_{2}\right]$ since they require more extensive discussion of the ergodic theory of hyperbolic flows than it seems to be appropriate for this paper.

Let $N$ be a compact $m$-dimensional manifold with negative curvature. Denote by $S N$ the unit tangent bundle over $N$ with the canonical projection $p: S N \rightarrow N$; that is, $v \in S N$ is a pair $(x, \xi)$ where $x \in N$ is a point, $\xi \in S_{x} N$ is a unit tangent vector to $N$ at $x$, and $p v=x$. The geodesic flow on $S N$ is the one parameter group of diffeomorphism $\phi_{t}: S N \rightarrow S N,-\infty<t<\infty$ such that $\phi_{t}(x, \xi)=(y, \eta)$ where $\operatorname{dist}(x, y)=t, y$ lies on the geodesic starting at $x$ with the speed vector $\xi$, and $\eta$ is the tangent vector to this geodesic at $y$. The geodesic flow preserves the Liouville measure whose volume element has the form $d x d \xi$ where $d x$ and $d \xi$ are the Riemannian volume elements 
in $N$ and $S_{x} N$, respectively. One also defines naturally a Riemannian metric on $S N$, the corresponding distance function will be denoted by $d(\cdot, \cdot)$. Next, we shall introduce local unstable and stable manifolds corresponding to the geodesic flow on the compact negatively curved manifold $N$ by

$$
W_{\varepsilon}^{s u}(v)=\left\{v^{\prime} \in S N: d\left(\phi_{t} v, \phi_{t} v^{\prime}\right) \leq \varepsilon e^{\varepsilon t} \text { for all } t \leq 0\right\},
$$

and

$$
W_{\varepsilon}^{S}(v)=\left\{v^{\prime} \in S N: d\left(\phi_{t} v, \phi_{t} v^{\prime}\right) \leq \varepsilon \text { for all } t \geq 0\right\},
$$

where $v \in S N$ and $\varepsilon>0$. The "su" stands for strongly unstable and " $s$ " stands for stable (sometimes the former is called just "unstable" and the latter "central-stable"). For $\varepsilon$ small enough $W_{\varepsilon}^{s u}(v)\left(\right.$ resp. $\left.W_{\varepsilon}^{s}(v)\right)$ is a $C^{1}(m-$ 1)-dimensional (resp. $m$-dimensional) disk embedded into $S N$ which varies continuously with $v$, and $W_{\varepsilon}^{s u}(v)$ and $W_{\varepsilon}^{s}(v)$ are transversal (see [A]).

For any $x \in M$ we define $\tilde{\mu}_{x}=\Phi_{x}^{-1} P(x, \cdot)$ which is a probability measure on $S_{x} M$. Since $N=M / \Gamma$ and the Brownian motion is invariant with respect to isometries $\gamma \in \Gamma$ then it is easy to see that $D \gamma \tilde{\mu}_{x}=\tilde{\mu}_{\gamma x}$ where $D \gamma$ is the differential of an isometry $\gamma: M \rightarrow M$ and we use the same notation for the action on points and measures on $S_{x} M$. This leads to the family of measures $\mu_{y}$ on $S_{y} N, y \in N$.

The main idea of the proof of Theorem 5.1 is the construction of a probability measure $\mu$ on $S N$ invariant with respect to the geodesic flow $\phi_{t}$ and such that its projections onto spheres $\left\{S_{y} N, y \in N\right\}$ along stable manifolds produce measures $\hat{\mu}_{y}, y \in N$, equivalent to measures $\mu_{y}, y \in N$, from the above family. Next, one employs Theorem $C^{\prime}$ from [LY] saying that the Hausdorff dimension of the $\phi_{t}$-invariant measure $\mu$ along the (strongly) unstable leaves equals $m-1$ if and only if the Pesin formula holds which would mean in the geodesic flow case that $\mu$ is the Liouville measure. Though the submanifolds $S_{y} N, y \in N$, are different from (strongly) unstable disks they are transversal to stable disks and this turns out to be sufficient. Now if $\mu$ is the Liouville measure then both $\hat{\mu}_{y}$ and $\mu_{y}, y \in N$ are equivalent to the Riemannian volume on $S_{v} N$. This would imply that measures from the harmonic and the geodesic classes are equivalent which in the two-dimensional case means by [Ka] and $\left[\mathrm{Le}_{1}\right]$ that the curvature is constant.

The rigorous proof uses the following facts:

(i) The $\phi_{t}$-invariant measure $\mu$ mentioned above is obtained in $\left[\mathrm{Le}_{2}\right]$ as the unique equilibrium state for certain Hölder continuous function $F$ on $S N$, i.e. $\mu$ maximizes the quantity $h_{m}\left(\phi_{1}\right)+\int F d m$ over all $\phi_{t}$-invariant probability measures $m$ on $S N$ where $h_{m}\left(\phi_{1}\right)$ denotes the entropy of $\phi_{1}$ with respect to $m$ (see $\left[\mathrm{B}_{1}\right.$ and $\left.\left.\mathrm{BR}\right]\right)$. The construction of $\mu$ resembles the construction of the socalled Sinai-Bowen-Ruelle measure which has conditional measures on unstable leaves equivalent to the volume there but, accordingly, we have to use the family of measures $\left\{\mu_{y}, \quad y \in N\right\}$ projected to unstable leaves in place of Lebesgue measures there. This leads to the function $F(v)$ which can be represented via 
the Martin kernel whose Hölder continuity (see [AS, Theorem 6.3]) implies the Hölder continuity of $F$. According to $\left[\mathrm{Le}_{2}\right]$ the measure $\mu$ described above being projected to the spheres $\left\{S_{y} N, y \in N\right\}$ produces measures which are equivalent to $\mu_{y}, y \in N$, i.e., for all $\varepsilon>0, y \in N, A \subset S_{y} N$

$$
\mu\left(\bigcup\left\{W_{\varepsilon}^{s}(v), v \in A\right\}\right)=\int_{A} G(y, v) \mu_{y}(d v) .
$$

Moreover one can choose the function $G$ in (5.4) to be positive and continuous on $S N$.

(ii) Suppose that $\mu$ is a $\phi_{t}$-invariant probability measure on $S N, \eta$ is a measurable partition of $S N$ such that $\eta(v)$ is a neighborhood of $v$ inside $W_{\varepsilon}^{s u}(v)$ for $\mu$-a.e. (almost every) $v \in S N$, and $v \rightarrow \mu_{v}$ is a family of regular conditional probabilities for $\mu$ associated with $\eta$. Then by Theorem $\mathrm{C}^{\prime}$ from [LY] there exists a number $\kappa, 0 \leq \kappa \leq m-1$, such that at $\mu$-a.e. $v$,

$$
\lim _{\varphi \rightarrow 0} \frac{\ln \mu_{v}\left(B^{s u}(v, \varphi)\right)}{\ln \varphi}=\kappa
$$

where $B^{s u}(v, \varphi)$ is the ball of radius $\varphi \leq \varepsilon$ inside $W_{\varepsilon}^{s u}(v)$ centered at $v$. Moreover $\kappa=m-1$ if and only if the Pesin formula holds for $\mu$, i.e. the entropy $h_{\mu}\left(\phi_{1}\right)$ is equal to the sum of positive Lyapunov exponents. But this happens if and only if $\mu$ is the Liouville measure since then $\mu$ must be the unique equilibrium state corresponding to the differential volume growth on unstable leaves (see [BR]).

(iii) At any point $v \in S N, S_{p v} N$ is transversal to $W_{\varepsilon}^{s}(v)$ with an angle bounded away from zero since both fields $T_{v} S_{p v} M$ and $T_{v} W_{\varepsilon}^{s}(v)$ are continuous and they have no common vectors.

From (iii) and the uniform continuity of $W_{\varepsilon}^{s u}$ it follows that there exists a constant $K>0$ such that

$$
B^{s u}\left(v, \frac{\varphi}{K}\right) \subset W_{\varepsilon}^{s u}(v) \cap\left(\bigcup_{u \in B(v, \varphi)} W_{\varepsilon}^{s}(u)\right) \subset B^{s u}(v, K \varphi),
$$

where $B(v, \varphi)$ is the ball of radius $\varphi$ inside $S_{p v} N$ centered at $v$.

(iv) It is known (see, for instance, $\left[\mathrm{B}_{2}\right]$ ) that there exist $\varepsilon, \delta>0$ small enough such that for each $v \in S N$ the map $z_{v}: W_{\varepsilon}^{s u}(v) \times W_{\varepsilon}^{s}(v) \rightarrow S N$ defined by

$$
z_{v}(a, b)=W_{\delta}^{s}(a) \cap W_{\delta}^{s u}(b)
$$

is a homeomorphism between the direct product $D^{m-1}(\varepsilon) \times D^{m}(\varepsilon)$ of $(m-1)$ dimensional and $m$-dimensional disks and a neighborhood $\mathscr{O}_{v}$ of $v$, called a rectangle. This homeomorphism is separately smooth in $a$ and $b$. Furthermore, let $\mu$ be an equilibrium state for a Hölder continuous function $F$. Consider a family $\mu_{u}, w \in \mathscr{O}_{v}$ of conditional measures for $\mu$ associated with the partition $\left\{W_{\varepsilon}^{s u}(b), b \in W_{\varepsilon}^{s}(v)\right\}$ of $\mathscr{O}_{v}$ where $\mu_{w}$ is a measure on $W_{\varepsilon}^{s u}(b), b \in W_{\varepsilon}^{s}(v)$ provided $w \in W_{\varepsilon}^{s u}(b)$. Let $\hat{\mu}_{b}, b \in W_{\varepsilon}^{s}(v)$ denote measures obtained from $\mu_{v}$ by projection along leaves $\left\{W_{\varepsilon}^{s}(a), a \in W_{\varepsilon}^{s u}(v)\right\}$ from 
$W_{\varepsilon}^{s u}(v)$ to $W_{\varepsilon}^{s u}(b)$. Then the measures $\mu_{b}$ and $\hat{\mu}_{b}, b \in W_{\varepsilon}^{s}(v)$ are equivalent with continuous densities which can be proved by a slight modification of methods in [BR] (see also [Si]). This means also that $z_{v}^{-1} \mu$ is equivalent with a continuous density to a product measure on $D^{n-1}(\varepsilon) \times D^{n}(\varepsilon)$.

Now the proof of Theorem 5.1 proceeds in the following way. Choose a finite set $\left\{v_{i}, i \in I\right\}$ of points from $S N$ so that $\bigcup_{i \in I} \mathscr{O}_{v_{1}}=S N$. Fix $i \in I$ and apply (ii) to the equilibrium state $\mu$ introduced in (i) and to the partition defined by $\left\{W_{\varepsilon}^{s u}(b), b \in W_{\varepsilon}^{s}\left(v_{i}\right)\right\}$. Then at $\mu$-a.e. $v$ we obtain (5.5) where $\mu_{v}$ is a family of conditional measures for $\mu$ associated with $\left\{W_{\varepsilon}^{s u}(b), b \in W_{\varepsilon}^{s}\left(v_{i}\right)\right\}$. By (iv) we can replace $\mu_{v}$ in (5.5) by the measures obtained from $\left.\mu\right|_{\mathscr{\sigma}_{v_{1}}}$ by projection along $\left\{W_{\varepsilon}^{s}(a), a \in W_{\varepsilon}^{s u}\left(v_{i}\right)\right\}$. By (5.6) we obtain the same limit if we project to the sphere $S_{p v} N$ and by (5.4) we finally can replace these projected measures by the measures $\mu_{p v}$ to obtain for $\mu$-a.e. $v \in S N$ that

$$
\lim _{\varphi \rightarrow 0} \frac{\ln \mu_{p v}(B(v, \varphi))}{\ln \varphi}=\kappa .
$$

Let $E$ be the set of points satisfying (5.7). We just proved that $\mu(E)=1$. On the other hand the set $E$ is made of stable manifolds since if $v$ and $v^{\prime}$ belong to the same stable manifold there exist $\zeta \in S(\infty)$ and points $\tilde{v}, \tilde{v}^{\prime} \in S M$ covering $v, v^{\prime} \in S N$, respectively, such that $\Phi_{p v} \tilde{v}=\Phi_{p v^{\prime}} v^{\prime}=\zeta$. In dimension 2 (and this is the only point we use that $m=2$ ) the maps $\Phi_{x}^{-1} \Phi_{y}: S_{y} M \rightarrow$ $S_{x} M, x, y \in M$, are continuously differentiable and, in particular, they are Lipschitz continuous (see [H, Lemma 1.43]) which implies

$$
\lim _{\varphi \rightarrow 0} \frac{\ln \mu_{p v}(B(v, \varphi))}{\ln \varphi}=\lim _{\varphi \rightarrow 0} \frac{\ln \mu_{p v^{\prime}}\left(B\left(v^{\prime}, \varphi\right)\right)}{\ln \varphi} .
$$

Since by (5.4), $\mu_{v}\left(E \cap S_{y} N\right)=1$ these yield (5.1).

By Theorem 3.1, $\kappa$ is positive. When $\kappa=1$ then $\mu$ is the Liouville measure by (ii), and so by (i) for $p \mu$-a.e. $y \in N$ the measure $\mu_{y}$ is equivalent to the Lebesgue measure on $S_{y} N$. The measures from the geodesic and the harmonic classes are then equivalent. By $[\mathrm{Ka}]$ or $\left[\mathrm{Le}_{1}\right]$, the curvature is constant in this case.

The last assertion concerning the constant curvature relying upon [Ka] and $\left[\mathrm{Le}_{1}\right]$ is known only for the two-dimensional case. The remaining argument uses $\operatorname{dim} N=2$ only to prove that the function

$$
\liminf _{\varphi \rightarrow 0} \frac{\ln \mu_{p v}(B(v, \varphi))}{\ln \varphi}
$$

is constant along stable manifolds which follows from the Lipschitz continuity of the map $\Phi_{x}^{-1} \Phi_{y}$ in this case. A. Katok pointed out to us that this map is known to be Lipschitz continuous (even $C^{1}$ ) also in certain multidimensional situations, for instance, when the negative curvature is $\frac{1}{4}$ pinched (see [HP]). Thus we have 
Theorem 5.2. Suppose that maps $\Phi_{x}^{-1} \Phi_{y}$ are Lipschitz continuous which holds in particular when all sectional curvatures of $N$ are pinched between $-a^{2}$ and $-\frac{1}{4} a^{2}$ for some $a \neq 0$. Then there exists a positive number $\kappa \leq 1$ such that (5.1) and (5.2) hold true for all points $x$ on the universal cover $M$ of $N$ and for $\Phi_{x}^{-1} P(x, \cdot)$-almost all $\xi \in S_{x} M$. Moreover $\kappa=\operatorname{dim} N-1$ if and only if the geodesic and the harmonic classes of measures coincide.

Remark 5.1. We would obtain Theorem 5.2 for the general case if we could prove that the dimensional function in the left-hand side of (5.7) is constant along stable manifolds. We would obtain the result only for a dense set of $y$ if we knew that the measures $\left\{\mu_{y}, y \in N\right\}$ are equivalent to the conditional measures of $\mu$ associated to the fibration $\left\{S_{y} N, y \in N\right\}$. But this latter property is not clear to us either. Remark also that in the two-dimensional case one does not need the full strength of Theorem $C^{\prime}$ from [LY] and a simpler argument would suffice.

From Theorem $\mathrm{C}^{\prime}$ of $[\mathrm{LY}]$ and the arguments above we obtain

Corollary 5.2. Suppose that $N$ is a compact connected Riemannian surface with negative curvature and $\mu$ is the invariant measure satisfying (5.4). Then

$$
\kappa=\lambda^{-1} h_{\mu}\left(\phi_{1}\right)
$$

where $\lambda$ is the corresponding Lyapunov exponent given by the formula

$$
\lambda=\inf _{t>0} \frac{1}{t} \int_{S N} \ln \left\|D_{v} \phi_{t}\right\| \mu(d v) .
$$

The entropy $h_{\mu}\left(\phi_{1}\right)$ and the Lyapunov exponent $\lambda$ for $\mu$ can also be expressed in terms of asymptotic growth rates along Brownian paths on $M$ (see $\left.\left[\mathrm{Le}_{2}\right]\right)$.

Remark 5.2. The above proof becomes a bit easier technically if one considers geodesic horospherical coordinates in place of geodesic polar (spherical) coordinates. The theory of [Ki] and $\S \S 1-4$ of this paper go through in this set up, as well. We arrive then to harmonic measures on the horosphere at infinity in place of the sphere at infinity. Projecting these measures to corresponding horospheres along orthogonal geodesics as at the beginning of the proof of Theorem 5.1 we arrive to a family of measures on (strongly) unstable leaves. The construction of the corresponding equilibrium state in (i) becomes easier then.

\section{REFERENCES}

[A] D. V. Anosov, Geodesic flows on closed Riemannian manifolds with negative curvature, Proc. Steklov Inst. Math. 90 (1967).

[AS] M. T. Anderson and R. Shoen, Positive harmonic functions on complete manifolds of negative curvature, Ann. of Math. 121 (1985), 429-461.

$\left[\mathrm{B}_{1}\right] \quad \mathrm{R}$. Bowen, Equilibrium states and the ergodic theory of Anosov diffeomorphisms, Lecture Notes in Math., vol. 470, Springer-Verlag, Berlin, 1985.

$\left[\mathrm{B}_{2}\right]$ - Symbolic dynamics for hyperbolic flows, Amer. J. Math. 95 (1973), 429-460. 
[BR] R. Bowen and D. Ruelle, The ergodic theory of Axiom- $A$ flows, Invent. Math. 29 (1975), 181-202.

[Ch] I. Chavel, Eigenvalues in Riemannian geometry, Academic Press, Orlando, 1984.

[CE] J. Cheeger and D. G. Ebin, Comparison theorems in Riemannian geometry, North-Holland, Amsterdam, 1975.

[Fr] A. Friedman, Stochastic differential equations and applications, vols. 1, 2, Academic Press, New York, 1975.

[H] E. Hopf, Statistik der geodätischen Linien in mannigfaltigkeiten negativer Krümmung, Ber. Verh. Sächs. Akad. Viss. Leipzig 91 (1939), 261-304.

[HP] M. Hirsch and C. Pugh, Smoothness of horocycle foliations, J. Differential Geom. 10 (1975), 225-238.

[IW] N. Ikeda and S. Watanabe, Stochastic differential equations and diffusion processes, NorthHolland, Kodansha, 1981.

[Ka] A. Katok, Four applications of conformal equivalence to geometry and dynamics, Ergodic Theory Dynamical Systems 8 * (1988), 139-159.

[Ki] Yu. Kifer, Brownian motion and positive harmonic functions on complete manifolds of nonpositive curvature, Pitman Research Notes in Math., vol. 150, Longman, Harlow, 1986, pp. 187-232.

[Le $]$ F. Ledrappier, Propriété de Poisson et courbure negative, C.R. Acad. Sci. Paris 305 (1987), 191-194.

$\left[\mathrm{Le}_{2}\right] \_$, Ergodic properties of the Brownian motion on covers of compact negatively curved manifolds, Bol. Soc. Brasil. Mat. (to appear).

[LY] F. Ledrappier and L.-S. Young, The metric entropy of diffeomorphisms, Part II: Relations between entropy, exponents and dimension, Ann. of Math. 122 (1985), 540-574.

[Mo] Y. Moser, On Harnack's theorem for elliptic differential equations, Comm. Pure Appl. Math. 14 (1961), 577-591.

[Se] J. Serrin, On the Harnack inequality for linear elliptic equations, J. Analyse Math. 4 (1954/56), 292-308.

[Si] Ya. G. Sinai, Gibbs measures in ergodic theory, Russian Math. Surveys 27 (1972), 21-69.

[Y] L.-S. Young, Dimension, entropy and Lyapunov exponents, Ergodic Theory Dynamical Systems 2 (1982), 109-129.

Institute of Mathematics, Hebrew University of Jerusalem, Jerusalem, Israel

Department of Mathematics, Cornell University, Ithaca, New York 14853

Laboratoire de Probabilités, Université Pierre et Marie Curie, Paris Vi, 4 Place Jussieu, 75230 Paris Cedex 05, France 